\title{
Arachidic Acid
}

National Cancer Institute

\section{Source}

National Cancer Institute. Arachidic Acid. NCI Thesaurus. Code C68321.

A saturated long-chain fatty acid with a 20-carbon backbone. Arachidic acid is found naturally as a minor component of peanut oil. 